\title{
La cavité buccale du chameau
}

\author{
par M.A.F. TAYEB, M. V.SC. \\ College of veterinary medicine, FouadI University, Giza (Egypt)
}

\author{
Traduction : P.-C. BLIN \\ Chef de Travaux d'Anatomie à l'École Nationale Vétérinaire d'Alfort
}

\section{INTRODUCTION}

Chauveau (1891) decrit les lèvres comme minces, très mobiles, la supérieure divisée par un profond sillon en deux parties mobiles indépendantes.

Un grand nombre de longues papilles coniques à pointe postérieure longe la muqueuse jugale de la commissure des lèvres à la première dent molaire. La couleur de ces papilles est noir uniforme. Le palais prósente une lògèrc crêtc saillante occupant ses deux tiers antérieurs.

La langue est très riche en papilles; elle est petite et présente des papilles foliacées.

LESBRE (1903) indique que les lèvres du chameau sont très mobiles; la lèvre inférieure est pointue; la supérieure est divisée par un sillon médian en deux parties.

En face interne, elles sont dépourvues de papilles. Les joues sont hérissées de longues papilles en odontoĩdes dirigées en arrière. Les deux tiers antérieurs du palais sont très étroits et il existe une crête médiane à leur surface. Le bourrelet incisif est semi-lunaire. La langue présente divers types de papilles; elle a deux cordons médians fibro-squelettiques et un double frein.

Le muscle stylo-glosse se rattache au stylo-hyal. Le basio-glosse est mince et le génio-glosse s'insère à la fois sur le basi-hyal et l'hypo-hyal.

LEESE (1927) rapporte que la lèvre supérieure est fendue, présente une grande liberté de mouvement et est poilue extérieurement.

La lèvre inférieure est épaisse et pendante.

Le bourrelet incisif est résistant et les papilles qui ornent la muqueuse des joues s'étendent jusqu'à la première molaire. Les antérieures sont coniques et dirigées vers l'arrière.

La langue présente six à sept papilles caliciformes.

L'objet de cette étude est la mise au point de l'étude de la cavité buccale du chameau.

\section{MATERTEL D'ÉTUDE ET MÉTHODE}

Les organes décrits dans cet article ont été prélevés aux abattoirs du Caire. Sept têtes de chameaux soudanais mâles et femelles ont été prélevées, après section en arrière de l'axis. La peau était intacte.

Les têtes furent injectées avec une solution de formol à $10 \%$ par l'artère carotide. Par ailleurs, les mêmes organes furent étudiés in situ sur des cadavres à l'abattoir même.

\section{LA CAVITÉ BUCCALE}

\section{Les lèvres.}

E.lles sont très souples et mobiles; l'inférieure est plutôt pointue et pendante et forme un menton bien développé.

La lèvre supérieure est deux fois plus épaisse que l'inférieure et la surplombe.

La lèvre supérieure est complètement divisée par un sillon tres profond ou philtrum en deux parties mobiles indépendantes. Chaque bord latéral de ce sillon est en continuité avec l'aile externe du naseau du côté correspondant.

Le nez se confond avec les lèvres dans son contour et ne forme pas de museau ou de groin chez le chameau.

Les deux lèvres sont recouvertes d 'une peau velue, souple et douce au toucher. Le duvet, de coloration grisâtre, s'étend aussi loin que le fait la face buccale ou interne des lèvres au niveau de laquelle il devient plus long et $s$ 'oriente vers la cavité buccale.

De longs poils tactiles dispersés se rencontrent à la face externe des lèvres et des naseaux. Il existe de nombreuses glandes labiales dans la lèvre supérieure. Elles sont peu nombreuses, petites, dispersées dans la lèvre inférieure. La muqueuse labiale est de coloration gris bleuté.

La cavité buccale affecte la forme d'une bouteille, étroite antérieurement et spacieuse postérieurement. Elle peut s'ouvrir largement par suite de la position plus reculée de la commissure des lèvres et de l'écartement plus important entre les attaches du buccinateur sur le maxillaire supérieur et sur le maxillaire inférieur, que chez les Équidés et les Bovidés.

\section{Les joues.}

Un trait caractéristique de la morphologie des joues chez le chameau est la présence d'une couche 
épaisse et ininterrompue de glandes buccales qui couvrent les deux côtés de la bouche, où l'on ne saurait distinguer de groupe supérieur et de groupe inférieur.

Elles s'étendent de la commissure des lèvres en avant jusqu'à la face interne de l'apophyse coronoïde approximativement, en arrière (contrairement à Chauveau et à Leese qui les font s'arrêter seulement à la première molaire). En haut et en bas, elles repoussent le buccinateur au-delà de ses attaches osseuses en formant des bords épais et rebondis, le supérieur étant le plus saillant.

En partie inférieure de la face externe du maxillaire inférieur, en regard même du masséter ou recouvrant légèrement son bord antérieur, se trouve la glande molaire inférieure, de contour triangulaire, s'étendant en avant, visible au travers du tendon semitransparent du buccinateur dans cette région. La glande mesure environ 6 à 8 centimètres de longueur, $2 \mathrm{~cm} .2$ à 5 centimètres de largeur et 2 centimètres d'épaisseur. Son long conduit excréteur s'ouvre par deux ou trois orifices au-dessous de la série des papilles.

La muqueuse qui borde les joues donne saillie à un massif touffu de papilles de dimensions et de formes diverses.

Ces papilles sont disposées en rangées longitudinales régulières simulant autant de scies placées les unes derrières les autres. La base épaisse et élargie de ces papilles est surmontée d'une ou de plusieurs extrémités libres pointues; de nombreuses papillos próscntent des denticules secondaires plus petits, issus de ces extrémités libres. Les plus longues papilles mesurent de 2 à $2 \mathrm{~cm}$. 5 environ et sonl étroitemenl cantonnées au pourtour de la commissure des lèvres.

Plus en arrière, leur taille diminue progressivement. Leurs extrémités varient dans leur direction; au voisinage de la commissure des lèvres ou à son pourtour, les papilles des séries inférieures sont orientées d'avant en arrière et de bas en haut; celles des séries supérieures sont orientées d'avant en arrière et de haut en bas. Postérieurement, toutes les papilles atteignent progressivement la position verticale, de sorte que les papilles des séries supérieures pointent de même que celles des séries inférieures vers la ligne moyenne qui les sépare.

Sur quelques sujets, des papilles mousses, arrondies, occupaient une ou deux rangées moyennes.

D'ordinaire, la couleur de ces papilles est bleu foncé, se nuançant quelquefois en rose pâle dans les séries postérieures.

\section{La langue.}

L'organe, blanc grisâtre, se déplace avec aisance; il a une longueur de 40 centimètres environ. La partie postérieure de la langue et sa racine s'infléchissent fortement; il en résulte une très profonde dépression en arrière de la racine de la langue, à sa jonction avec le plancher pharyngien.

Par suite de l'absence du prolongement entoglosse de l'os hyoide, la langue ne se rattache pas directement à cet os.

Elle présente une pointe ou apex aplatie, arrondie, recouverte de fines papilles fongiformes.

En arrière de sa pointe, l'organe devient plus étroit, mais plus épais dans son diamètre dorsoventral, de sorte qu'il prend un aspect colleté.

La partie antérieure du corps de la langue est convexe, recouverte de fortes papilles cornées filiformes, toutefois moins développées que chez le boeuf.

La partie postérieure est nettement délimitée par rapport à la partie antérieure : elle présente un massif compact de papilles arrondies, rappelant les papilles lenticulaires du bœuf, mais plus étendues, formant une protubérance triangulaire à sommet antérieur.

En arrière, ces papilles sont remplacées sur une distance de 6 centimètres environ par un groupe de papilles arrondies, à orientation antérieure. Un groupe postérieur présente ce même type de papilles mais avec une orientation postérieure.

De chaque côté existe une série de cinq à sept papilles caliciformes dont le diamètre est supérieur à celui des mêmes papilles chez le cheval.

En arrière des papilles caliciformes se trouvent, en rangs compacts, d'épaisses papilles en massue qui occupent la base de la langue; dans l'espace médian qui sépare celles d'un côté de celles de l'autre, existe une plage arrondie faite de tissu lymphoide correspondant aux amygdales.

Il n'y a pas de cordons fibro-squelettiques de la langue, comme le soutient Lesbre. Les papilles foliées font défaut. Le double frein de la langue est facile à mettre en évidence. Les muscles extrinsèques de la langue sont plus développés que chez le cheval.

Le styloglosse est allongé et épais; il s'insère sur le stylohyal juste au-dessus du cartilage de synchondrose qui réunit le stylohyal au cératohyal (chez le chameau, le cératohyal est bien développé et mesure en longueur, les deux tiers environ du stylohyal). Le grand hyoglosse (ou basio-glosse) est un muscle épais, bien développé s'attachant par un tendon large et fort sur le corps et les cornes thyroidiennes de l'hyoïde recouvrant le kérato-hyoïdien qu'il masque complètement. Il prend également une attache mince et faible sur le cératohyal.

Le génioglosse est un muscle très épais; un mince faisceau de fibres, ayant environ 1 à 2 millimètres d'épaisseur, se détache de la partie inférieure du muscle pour s'insérer șur le corps de l'hyoïde. 
Figure $v$.

1. Face interne ou buccale des joues.

v. Glande molaire inferieure. -- O. Orifice du canal de Sténon s'ouvrant sur une papille aplatie et arrondie. - P. Diverses formes de papilles. 0. Lèvre supérieure. - L'. Lèvre inférieure (Remarquez la disposition des poils qui s'étendent jusqu'à la face buccale des lèvres et sont orientés vers la cavité buccale.) - D. Bord supérieur.

2. Vue externe de la couche épaisse et continue des glandes buccales.

v. Vue latérale de la glande molaire inférieure. -- L'. Lèvre inférieure. - U. Lèvre supérieure. D. Bord supérieur. - P. Face externe de la parotide. - B'. Sa base; $\mathbf{X}$. Son sommet. - M. Face ezterne de la glande sous-maxillaire. -- B. Sa base ou extrémité supérieure. - $\mathbf{-}$. Bord antérieur. - A'. Son sommet. - S. Ganglion Iymphatique rétropharyngien; observez la gouttière de sa face interne, à travers laquelle passe l'artère carotide. - L. Ganglion sous-maxillaire. - TT. Sa base postérieure convere.

M. Glande sous-maxillaire.

B. Extrémité supérieure. - A'. Aper. --

A. Bord antérieur.

P. Parotide.

B'. Base. - X. Apex.

S. Face externe d'un ganglion rétropharyngien.

T. Ganglion sous-maxillaire.

L. Base.

L'. Lèvre inférieure.

ס. Lèvre supérieure.

G. Glandes buccales

P. Papilles cornées.

O. Ouverture du canal de Sténon.

V'. Glande molaire inférieure.

1. Face interne de la joue.

2. Face externe de la joue.

D. Bord dorsal.

V. Bord ventral.

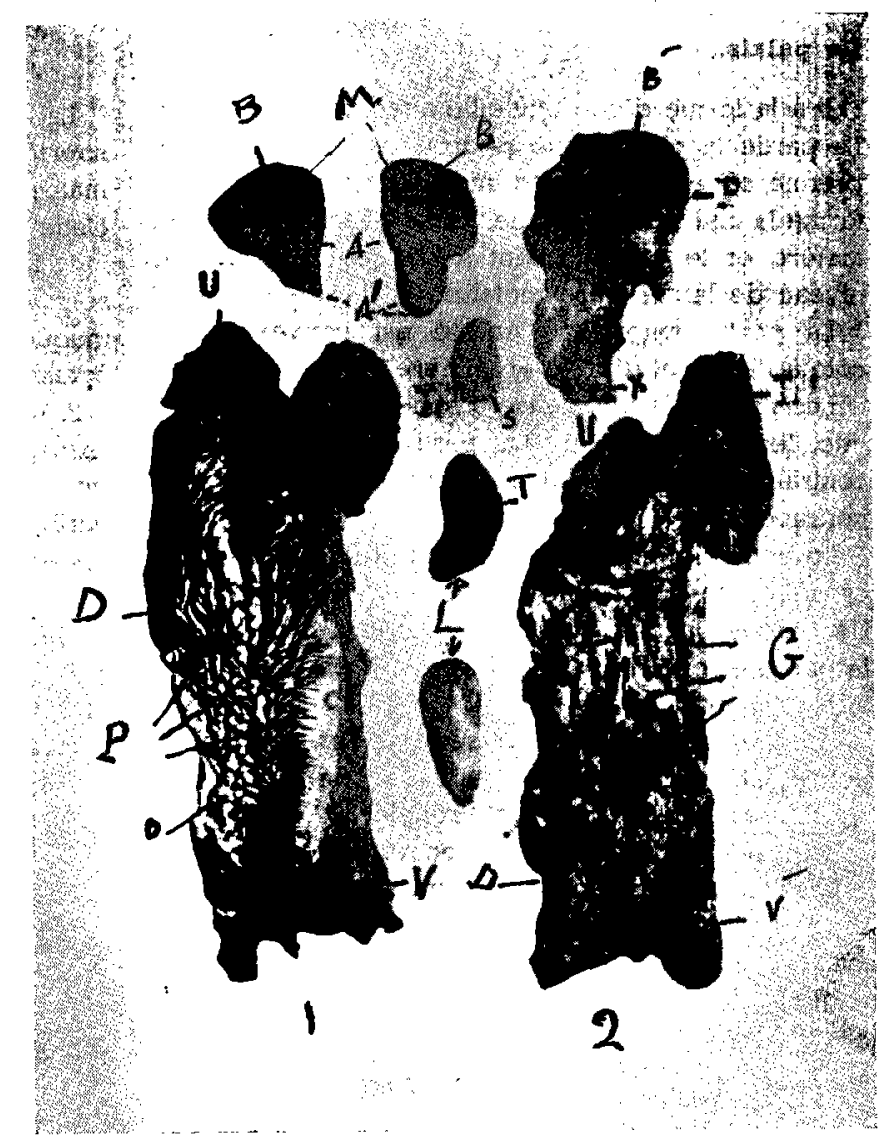

Figure VI.

1. Le large bourrelet dentaire en forme de croissant.

2. La portion antérieure étroite du palais (observez la créte longitudinale centrale et les crêtes transversales en dents de scie, rejoignant la'crête centrale sous divers angles).

3. La portion postérieure, spacieuse; lisse et excavée du palais.

4. Portion de la poche palatine (palais mou).

5. La pointe da la langue, recouverte de papilles fongiformes.

6. La partie étroite et colletée de la langue.

7. Massif de papilles mousses arrondies (papilles lenticulaires de la protubérance dorsale de la langue du bceuf) formant une saillie triangulsire; son sommet antérieur.

8. Papilles caliciformes.

- 9. Papilles arrondies, à orientation antérieure, puis postérieure.

10. Amygdales linguales.

11. Papilles en massue, dirigées en arrière.

12. Prolonde dépression sise à la base dé la langue.

13. Epiglotte.

14. Repli glosso-épiglottique saillant. 


\section{Le palais.}

Il a la forme d'une bouteille avec un long goulot. La partie la plus étroite se trouve située entre les premières et deuxièmes molaires supérieures et forme là une sorte d'isthme. Puis l'isthme s'évase en arrière et le palais atteint sa largeur maxima au niveau de la dernière molaire.

La partie antérieure étroite est convexe d'un côté à l'autre et d'avant cn arrièrc.

Les plis de la muqueuse à son niveau constituent une légère élévation. La partie postérieure; au conlraire, fait transition insensible et apparaît très concave.

Le bourrelet incisif est résistant et forme un large croissant de coloration grisâtre ou jaune grisâtre. Un raphé central bien délimité occupe toute la partie étroite du palais et le divise en deux territoires égaux.

Les crêtes transversales sont, elles aussi, bien nettes; elles présentent de légères indentations ou, au contraire, sont foxtement crénelées.

Les crêtes ont une orientation variable.

Les crêtes antérieurẹs rencontrent le raphé médian sous un angle aigu, dont le sommet est dirigé en avant, les crêtes moyennes forment avec lui un angle droit; enfin, les crêtes postérieures contribuent à former avec le raphé un angle à sommet dirigé en arrière. La coloration du palais est bleu foncé, mais chez quelques sujets la coloration bleu intense se nuance de teintes jaune pâle ou gris lavé au niveau de la partie postérieure lisse et concave du palais.

\section{RÉSUMÉ}

La cavité buccale du chameau a été étudiée sur sept têtes injectées provenant de chameaux soudanais mâles et femelles, prélevées aux abattoirs du Caire. Cette même cavité fut également étudiée in situ.

1. Les lèvres sont souples et très mobiles. Elles sont recouvertes de poil qui s'étend jusqu'à la muqueuse buccale. La lèvre supérieure est plus épaisse et surplombe l'inférieure.

2. La lèvre supérieure est divisée en deux parties susceptibles de se mouvoir indépendamment l'une de l'autre par un. profond sillon médian dont les bords sont en continuité avec les ailes externes des naseaux.

3. Le nez se confond avec la lèvre supérieure; le chameau ne présente ni mufle, ni groin.

4. La face supérieure de la langue présente un grand nombre de papilles de forme et de dimensions différentes. Le corps et la racine de l'organe s'infléchissent brusquement pour constituer une profonde dépression avec le plancher pharyngien.

5. Il existe un double frein lingual, mais les cordons fibro-squelettiques et les papilles foliees font défaut.

6. Le palais est long et étroit en sa partie antérieure. Le bourrelet incisif forme un large croissant.

\section{BIBLIOGRAPHIE}

1. CHAUVEAU $\left(A_{\text {. }}\right)$. - The comparative anatomy of the domes. ticated animals. Second english edition, published by J. and F. Churchill Londón II, New Burlington Street. I89I.

2. LESBLE (M.F.X.). - Recherches anatomiques sur les Cámé. lidés. Extr, from Archives du Museum d'Hist. Nat. Lyon 1903.

3. LEESE (A.-S.). - A treatise on the one-humped camel. Published and printed by Haynes and Sons, Maiden Lane. Stamford, Lincolnshire, 1927. 